\title{
Time course of gustatory adaptation
}

\author{
JANNEANE F. GENT and DONALD H. McBURNEY \\ University of Pittsburgh, Pittsburgh, Pennsylvania 15260
}

\begin{abstract}
Subjects rated the taste of solution-soaked filter paper over time. Adaptation was complete for all compounds at most concentrations. The course was exponential and the same for all conditions. Adaptation was not the result of stimulus loss. The filter-paper technique allows a greater stability of stimulus than other techniques, hence complete adaptation.
\end{abstract}

The time course of adaptation and its completeness have been difficult to demonstrate in the taste system. Abrahams, Krakauer, and Dallenbach (1937; Krakauer \& Dallenbach, 1937) were the first to demonstrate complete adaptation in taste. They used a method designed to flow a solution of constant concentration through the entire mouth. While this method did reduce salivary dilution of the solution, the elimination of tongue movements was left to the subject. It was extremely difficult to maintain a constant stimulation of a particular receptor population using this "Cornell gustometer." Cases in which complete adaptation were not observed were attributed to the failure of the subjects to eliminate tongue movements. Despite the difficulty of the subject's task, Abrahams et al. were able to measure time to complete adaptation for compounds representing each of the four qualities.

Meiselman (1968) made direct psychophysical measurements of the time course of adaptation and found that magnitude estimates of sensation intensity steadily declined although, contrary to Abrahams et al., complete adaptation did not take place. This could probably be attributed to the method of stimulus delivery, repeated sip and spit, which does not allow for control of tongue movements or salivary dilution. Meiselman and DuBose (1976) also investigated the possibility that the completeness of adaptation depends not only on the method of stimulus presentation but also on the task instructions given to the subject. They found, however, that instructional set failed to affect the outcome of their adaptation experiments, and once again, contrary to the earlier Abrahams et al. results, the majority of the subjects did not show complete adaptation.

Bujas (1953), using a flow chamber that rested on the front of the tongue, measured the time course of adaptation to $\mathrm{NaCl}$ with a taste intensity matching procedure. The matched concentrations declined roughly exponentially over time, although it is not possible, given his data, to say what the function describing sensation magnitude vs. adaptation time

Supported by NIH Grant NS 07873. would be. Complete adaptation was observed, perhaps because this method of stimulation allows for control over salivary dilution and more control over tongue movements than the sip and spit method, for example.

Studies using a matching procedure to trace the time course of $\mathrm{NaCl}$ adaptation have also been done in this laboratory (Shick, 1972; McBurney \& Gent, Note 1). We tried to combine the gravity flow method and apparatus for accurately controlling stimulus duration, used successfully in previous experiments (e.g., McBurney, 1976), with a device to hold the extended tongue relatively immobile. All of our tongue chambers were designed so that the base of the extended tongue rested against the solid bottom of a Plexiglas chamber while the top rested against partitions dividing the tongue into three areas. Adapting solution flowed to one of the side areas, a continuous water rinse flowed to the center, and the matching solution flowed to the other side. Each subject was requested to hold his tongue extended and still, which proved to be very uncomfortable and almost impossible. As a result, subjects found the matching task extremely difficult, which in turn made it difficult to observe the process of adaptation.

All of the results reviewed above suggest that the critical variable in accounting for differences among experiments is the control of effective stimulus concentration. McBurney (1976) investigated the response of the taste system to a changing stimulus using the techniques of linear systems analysis. The system proved to be even more sensitive than indicated by previous research. The threshold for detecting a change in concentration was measured using a stimulus with a sinusoidally varying concentration. At the lowest frequency studied, $.001 \mathrm{~Hz}$, a very slowly changing stimulus, the minimum detectable modulation (change in concentration relative to the average concentration) was $0.1 \% / \mathrm{sec}$. These results imply that unless the method used to study adaptation can assure a constant state of stimulation, the adaptation process will be difficult, is not impossible, to observe. These results explain our failure (McBurney \& Clendenning, Note 2) to replicate some results of Békésy (1965). We put a slide valve of the type used 
in the temporal modulation work under the control of the subject by means of a manually controlled gear. The intent was to have the subject increase the concentration of $\mathrm{NaCl}$ from zero at a rate that allowed adaptation to take place such that the subject never tasted the salt. The subjects found that it was impossible to advance the gear without producing a salty sensation once the concentration exceeded some value near threshold.

Thus, in light of the extreme temporal modulation sensitivity of the taste system, the successful study of the adaptation process can occur only if the stimulation method used is able to deliver a more constant stimulus to a specified receptor area than previous methods. One candidate for a method of immobile stimulus delivery is a small piece of filter paper soaked in solution and placed on a preselected location of the tongue. Collings (1974) and Hara (1955) have used this method of stimulus delivery, although not for the purposes of studying adaptation.

The present experiment shows that adaptation is an exponential process and is complete.

\section{METHOD}

\section{Subjects}

Twelve University of Pittsburgh students, uninformed as to the purpose or expected outcome of the experiment, served as subjects.

\section{Solutions}

Stimuli were solutions of $\mathrm{NaCl}(0.1,0.32,1.0 \mathrm{M})$, citric acid $(0.01,0.032,0.1 \mathrm{M})$, quinine sulfate $\left(\mathrm{QSO}_{4}\right)(0.000032,0.0001$, $0.00032 \mathrm{M})$, and sucrose $(0.1,0.32,1.0 \mathrm{M})$. $\mathrm{NaCl}$ and citric acid were reagent grade; $\mathrm{QSO}_{4}$ U.S.P.; and sucrose, commercial.

\section{Psychophysical Method}

Each subject was given practice in using magnitude estimation by rating lengths of multicolored lines. The subject was asked to assign any number to the total length of the first line he was shown, then to rate the lengths of all other lines in proportion to the first line. In addition, the subject was asked to divide his overall estimate of the total length of each line to indicate the proportion of each line that was a particular color. The subject then rated the intensity of each solution using nonmodulus magnitude estimation. In addition to rating the overall sensation magnitude, the subject divided each estimate among the four qualities and an "other" category for any nongustatory sensations (e.g., "pain," “"burning," "stinging," etc.)

\section{Procedure}

Stimulus delivery was by means of a small, circular piece of filter paper (13 mm in diameter) held with forceps. The filter paper was soaked in solution and drained of excess solution by tapping the edge against a paper towel. During the experimental session, the stimuli were placed exclusively on the left $(\mathrm{N}=6)$ or the right $(\mathrm{N}=6)$ anterior dorsal surface of the subject's tongue.

Before beginning the experimental trials, each subject was presented with samples of filter paper soaked in water and the middle concentration of each of the four compounds. Prior to each experimental trial, the subject rinsed his mouth with deionized water, then patted the excess moisture off of the front of his extended tongue with a paper towel. Subjects were instructed to hold their tongues comfortably extended, to try not to wiggle their tongues, and to try to keep saliva off of the filter paper. The subject was instructed to his his first magnitude estimate of the intensity of the solution 5 to $10 \mathrm{sec}$ after stimulus placement. The experimenter started a timer as the stimulus was placed on the subject's tongue. At $15-\mathrm{sec}$ intervals, the experimenter gave a verbal signal to the subject, who then recorded his current estimate of the sensation magnitude on the answer sheet. The experimenter continued to signal the subject for $3 \mathrm{~min}$. A trial was terminated before $3 \mathrm{~min}$ if the subject recorded two zero taste sensation estimates in a row.

Each subject participated in one approximately $1 \frac{1 / 2}{2} \mathrm{~h}$ session, during which each of the 12 test solutions was presented twice, once during the first half of the session and once during the second half. The order of presentation of the 12 solutions was random within each half.

\section{Control Experiments}

Before attempting to use the filter paper to study the time course of adaptation, several control experiments were conducted to investigate the possible loss of concentration over time, loss of solution over time, and possible effects of the rate of diffusion. The conductivity of the solution from the experimental filter paper soaked in $1.0 \mathrm{M} \mathrm{NaCl}$ was measured immediately after soaking and again after $2 \mathrm{~min}$ on the tongue, and compared to the conductivity of $\mathrm{NaCl}$ solutions of known molarity. It was found that the change in concentration of the solution on the filter paper over time was negligible.

The average piece of filter paper contained $.02 \mathrm{ml}$ of solution at the time it was placed on the control subject's tongue. The average loss of solution after $2 \mathrm{~min}$ on the tongue was $14 \%$. However, the loss of solution was probably the result of fluid left behind when the filter paper was removed from the tongue, because the same amount was lost whether the paper remained on the tongue for $25 \mathrm{sec}$ or $2 \mathrm{~min}$. In addition, solution was added to the solution-soaked filter paper already on the tongue by means of a capillary tube and wick arrangement. The four control subjects recorded direct magnitude estimates of the stimulus intensity every $15 \mathrm{sec}$, both with and without solution replacement. All four subjects reported a "flowing" sensation as solution was added to the filter paper, but none experienced any return or increase in intensity following careful solution replacement. Thus, replacing solution had no effect on the subsequent loss of sensation. It should be noted that, for all subjects, sensation returned only if the solution flowed off the edge of the filter paper as it was being replaced.

To test for the possible effects of rate of diffusion on the adaptation process, two control subjects tasted $\mathrm{NaCl}$ in water and $\mathrm{NaCl}$ in $10 \%$ and $30 \%$ solutions of polyethelene glycol, a tasteless compound that is substantially more viscous than water. There were no differences in the time to complete adaptation. This implies that the velocity of the mass transfer in solution is rapid compared to the speed of the process that results in adaptation (Weisz, 1973; Cussler, Note 3).

\section{RESULTS}

The data for each subject were rescaled so that the average magnitude estimate of total taste intensity for the initial rating was equal to 10 . The median estimates (based on the 24 ratings for each 15 -sec interval) of total intensity minus any "other" nongustatory ratings are shown in Figures 1-4. The initial median magnitude estimate of each solution is plotted at $t=6 \mathrm{sec}$ because this approximates time of rating relative to time of stimulus placement $(\mathrm{t}=0)$.

The median magnitude estimate of the intensity over time of all compounds except the strongest concentration of citric acid falls to zero in $\leqslant 120 \mathrm{sec}$. 


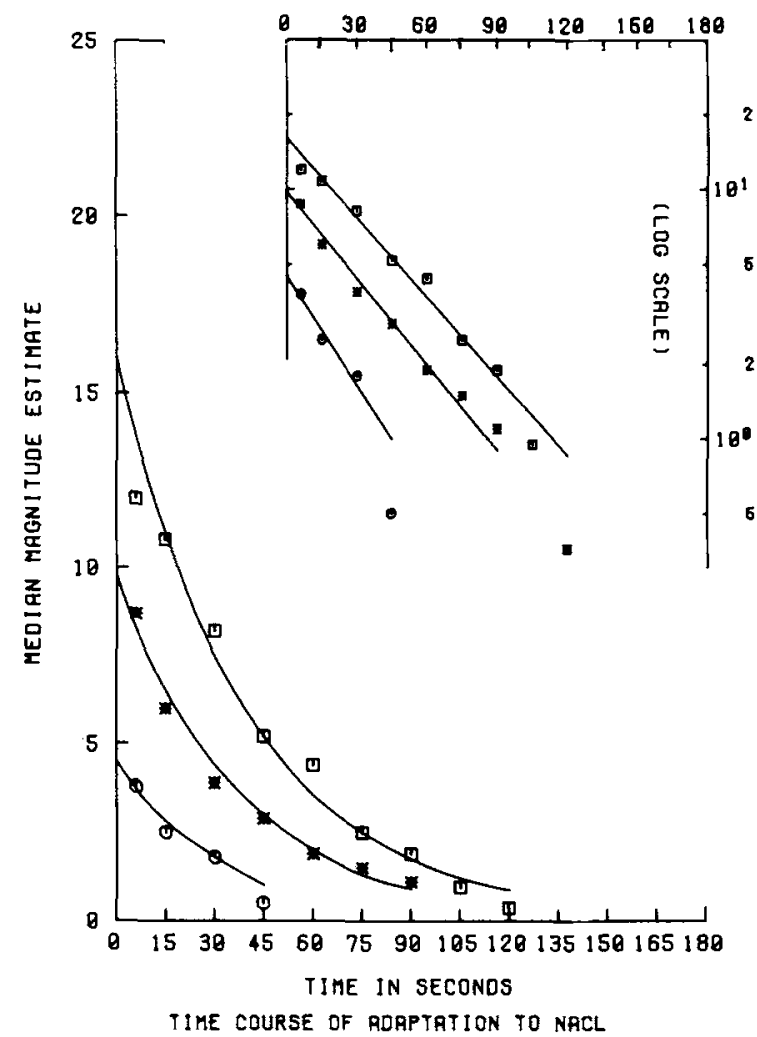

Figure 1. Median magnitude estimate of total taste intensity at 15-sec intervals for strong $(\square)$, medium $\left({ }^{*}\right)$, and weak $(O)$ concentrations of the adapting solution.

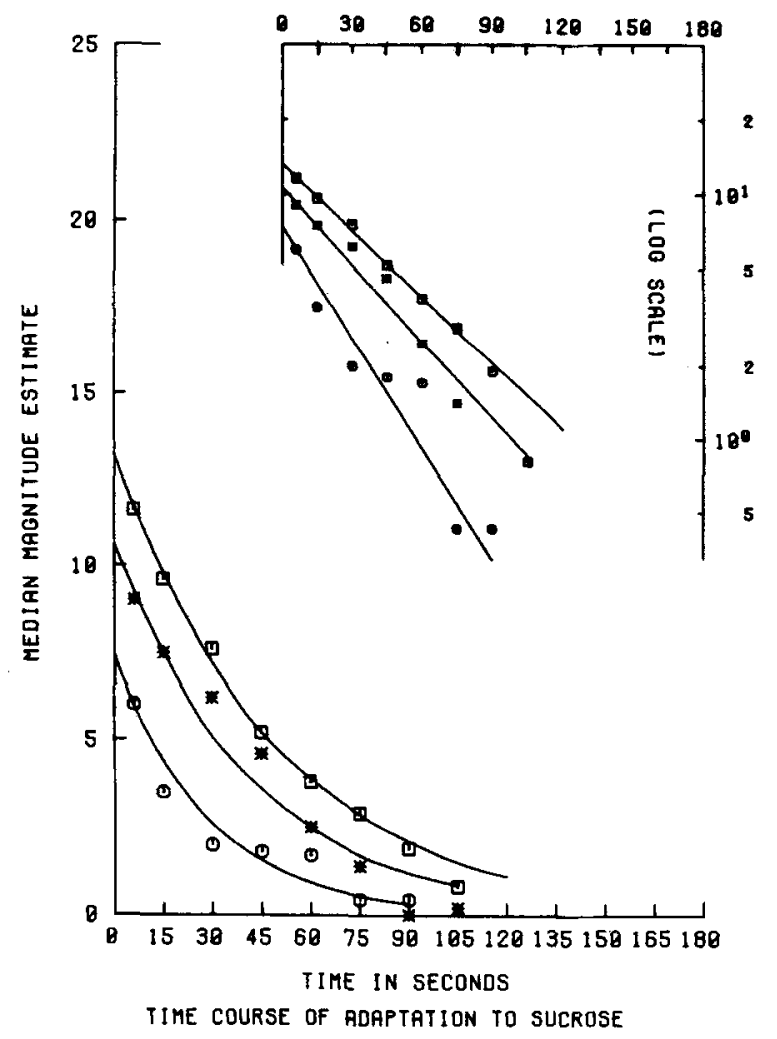

Figure 2. Same as Figure 1, for sucrose.
Furthermore, the time course of this decay is exponential and the same for all compounds. There was no significant difference between compounds in the slopes of the lines shown in the insets of Figures 1-4. In addition, no significant difference was found between the slopes representing the strong and medium concentrations within each compound. The consistently steeper slope for the weakest concentration was not considered reliable, since, in every case, adaptation to the weakest concentration took place in $\leqslant 90 \mathrm{sec}$. This resulted in fewer data points with which to estimate the decay function.

The function relating response magnitude to concentration and duration of stimulation can be written as follows:

$$
R=k S^{n} e^{-t / \tau} \text {, }
$$

where $\mathrm{R}=$ response magnitude; $\mathrm{k}=$ constant; $\mathrm{S}=$ stimulus concentration; $\mathrm{n}=$ exponent of the psychophysical function relating sensation magnitude to stimulus concentration (the following exponents were found: 0.50 for $\mathrm{NaCl} ; 0.25$ for sucrose; 0.78 for $\mathrm{QSO}_{4}$; and 0.60 for citric acid); $\mathrm{t}=$ duration of stimulation in seconds; and $\tau=$ time constant of decay (time it takes sensation to reach $1 / \mathrm{e}$ of its initial value) (in this case, $\tau=40 \mathrm{sec}$ or $-1 / \tau=$ $-.025)$.

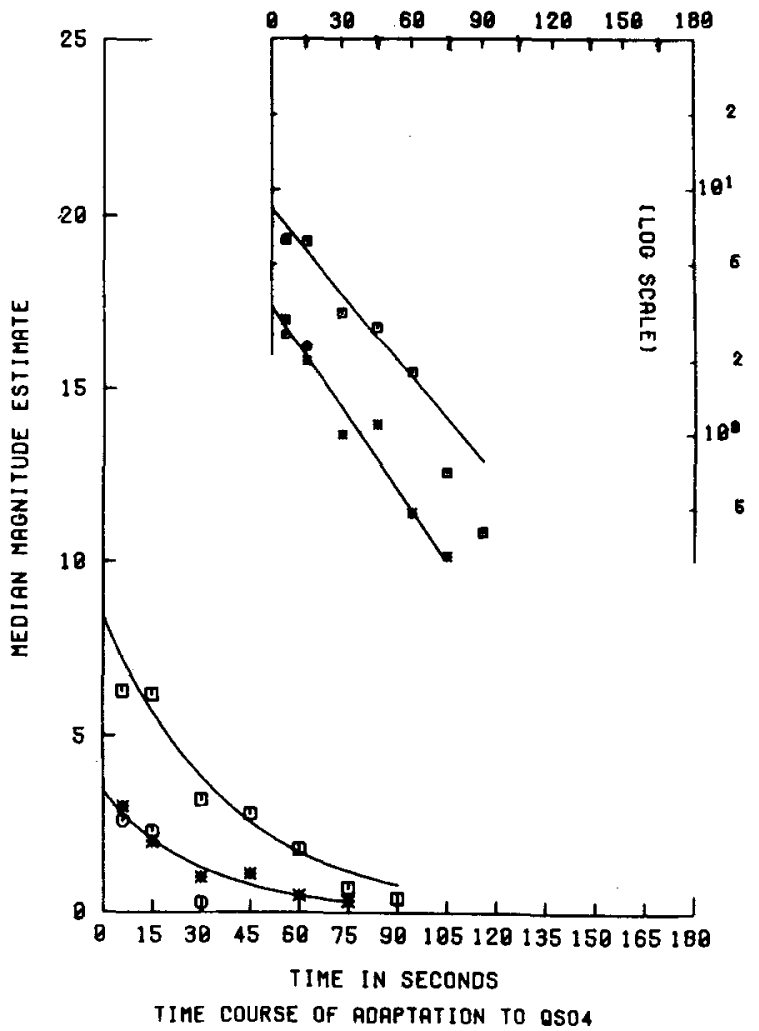

Figure 3. Same as Figure 1, for quinine sulphate. 


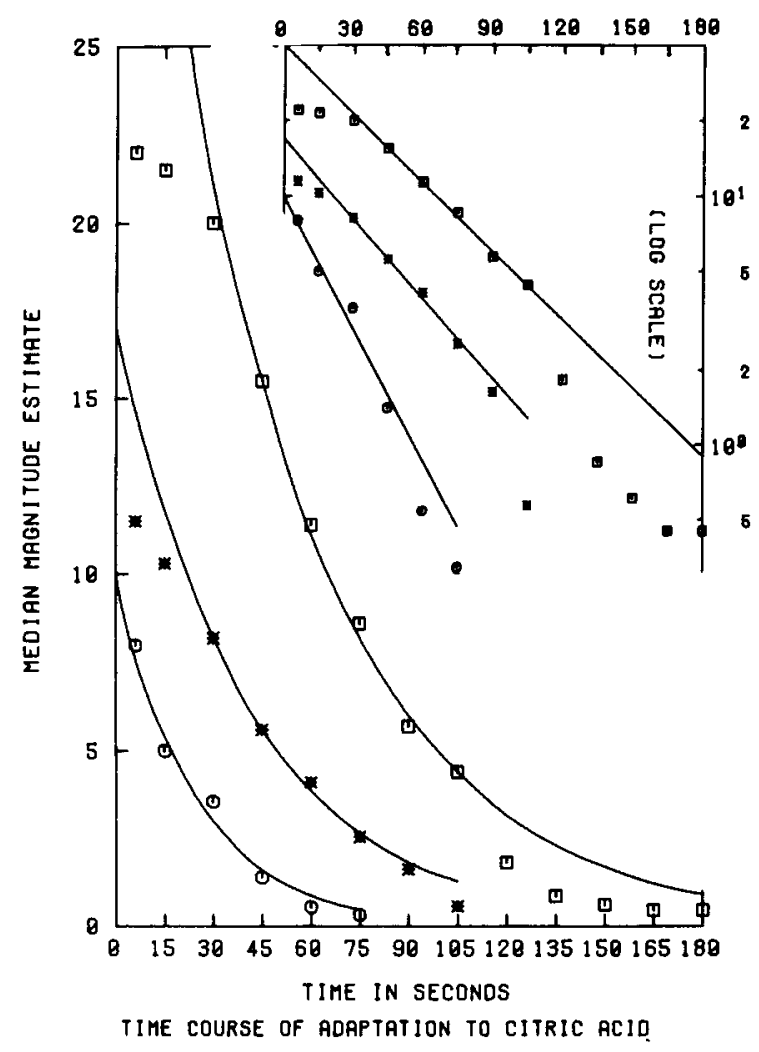

Figure 4. Same as Figure 1, for citric acid.

More than half of the subjects adapted completely to both presentations of all but the strongest concentrations of $\mathrm{NaCl}, \mathrm{QSO}_{4}$, and citric acid. In fact, 9 of the 12 subjects adapted completely to at least one presentation of at least 11 solutions. Of the three subjects who consistently failed to adapt completely within the $3 \mathrm{~min}$, two did experience a slowly declining sensation for all but two solution presentations (both presentations of the strongest $\mathrm{NaCl}$ for one subject and the first presentations of the two weakest $\mathrm{QSO}_{4}$ solutions for the other subject). The third subject only experienced a consistent, slowly declining sensation for the sucrose solutions. She experienced a slowly increasing sensation for the citric acid solutions perhaps due to an increasing "painful" sensation that she was unable to completely separate from the "taste" sensation. Her data for the $\mathrm{NaCl}$ and $\mathrm{QSO}_{4}$ solutions were erratic.

\section{DISCUSSION}

It is clear from the remarkable temporal modulation sensitivity of the taste system (McBurney, 1976) that complete adaptation may fail to take place in the presence of a stimulus of changing concentration. Changes in concentration occur unavoidably with most previously used methods of stimulation, especially sip and spit, and it is likely that no further explanation is necessary to account for the lack of complete adaptation observed by Meiselman (1968; Meiselman \& DuBose, 1976). In addition, it is obvious from temporal modulation sensitivity functions that a stimulus of regularly changing concentration such as a pulsatile stimulus (cf. Meiselman \& Halpern, 1973) will also prevent adaptation from taking place.

Completeness in adaptation is not necessarily the case in every sense (cf. Marks, 1974), but there have been studies in addition to Abrahams et al. (1937) indicating that complete adaptation does occur in taste. For example, the fact that the threshold after adaptation lies just above the adapting concentration (Hahn, 1934; McBurney \& Pfaffmann, 1963) indicates that the adapting stimulus has become tasteless. In fact, McBurney and Pfaffmann (1963) demonstrated what is the most obvious natural occurrence of complete adaptation in taste: the threshold for $\mathrm{NaCl}$ lies just above the salivary sodium level, thus explaining why saliva is tasteless. Complete adaptation has also been found in studies using stronger solutions than were used in the threshold experiments (Bartoshuk, 1968; Bartoshuk, McBurney, \& Pfaffmann, 1964).

The decay functions describing the adaptation process are remarkably similar for every compound, yet the psychophysical power functions relating magnitude intensity to stimulus concentration are all different. The taste system does not seem to be unique in this independence of stimulus quality and intensity from the adaptation process. Cross-modality matching procedures have been used to chart the time course of olfactory adaptation (Cain, 1974; Ekman, Berglund, Berglund, \& Lindvall, 1967). Ekman et al. (1967) found that an exponential function described their data quite well and that the rate of adaptation to hydrogen sulfide in air (their experimental stimulus) seemed to be independent of concentration. Cain (1974) used several different compounds having different psychophysical functions and also found that adaptation could be described by the same exponential function.

The similarity of the adaptation functions among qualities and the differences in the temporal modulation sensitivity functions for the same qualities implies that the two processes are distinct. It appears that adaptation is a peripheral process in human taste, whereas temporal summation involves central as well as peripheral mechanisms. Diamant, Oakley, Ström, Wells, and Zotterman (1965) found that the decrease in the response of the human chorda tympani to spontaneous level paralleled the psychophysical decline in the taste sensation, which indicates that adaptation is probably a peripheral process. On the other hand, temporal summation in the chorda tym- 
pani of the rat is complete within $100 \mathrm{msec}$, whereas the sensation in the human grows for up to about $10 \mathrm{sec}$, suggesting that central processes are probably involved.

\section{REFERENCES NOTES}

1. McBurney, D. H., \& Gent, J. F. Unpublished data, University of Pittsburgh, 1976.

2. McBurney, D. H., \& Clendenning, K. Unpublished observations, University of Pittsburgh, 1973.

3. Cussler, E. L. Personal communication, October 1977.

\section{REFERENCES}

Abrahams, H., Krakauer, D., \& Dallenbach, K. M. Gustatory adaptation to salt. American Journal of Psychology, 1937, 49, 462-469.

Bartoshuk, L. M. Water taste in man. Perception \& Psychophysics, 1968, 3, 69.72.

Bartoshuk, L. M., McBurney, D. H., \& Pfaffmann, C. Taste of sodium chloride solutions after adaptation to sodium chloride: Implications for the "water taste." Science, 1964, 143, 967-968.

BÉKÉSY, G. v. The effect of adaptation on the taste threshold observed with a semiautomatic gustometer. Journal of General Physiology, 1965, 48, 481-488.

Busas, A. L'adaptation gustative et son méchanisme. Acta Instituti Psychologici Universitatis Zagrebensis, 1953, 17, 1-11.

CaIn, W. S. Perception of odor intensity and the time-course of olfactory adaptation. American Society of Heating, Refrig. erating and Air-Conditioning Engineers Transactions, 1974, 80, 53.75.

Collings, V. B. Human taste response as a function of locus of stimulation on the tongue and soft palate. Perception \& Psychophysics, 1974, 16, 169-174.

Diamant, H., Oakiey, B., Ström, L., Wells, C., \& ZOTTERMAN, Y. A comparison of neural and psychophysical responses to taste stimuli in man. Acta Physiologica Scandinavica, 1965, 64, 67-74.

Ekman, G., Berglund, B., Berglund, U., \& Lindvall, T. Perceived intensity of odor as a function of time of adaptation. Scandinavian Journal of Psychology, 1967, 8, 177-186.

HaHN, H. Die Adaptation des Geschmackssinnes. Zeitschrift für Sinnesphysiologie, 1934, 65, 105-143.

HarA, S. Interrelationships among stimulus intensity, stimulated area and reaction time in the human gustatory sensation. Bulletin of Tokyo Medical and Dental University, 1955, 2, 147-158.

Krakauer, D., \& Dallenbach, K. M. Gustatory adaptation to sweet, sour, and bitter. American Journal of Psychology, 1937, 49, 469-475.

MARks, L. E. Sensory processes: The new psychophysics. New York: Academic Press, 1974.

McBurney, D. H. Temporal properties of the human taste system. Sensory Processes, 1976, 1, 150-162.

McBurney, D. H., \& Pfaffmans, C. Gustatory adaptation to saliva and sodium chloride. Journal of Experimental Psychology, $1963,65,523-529$.

Meiselman, H. L. Magnitude estimations of the course of gustatory adaptation. Perception \& Psychophysics, 1968, 4, 193-196.

Meiselman, H. L., \& DuBose, C. N. Failure of instructional set to affect completeness of taste adaptation. Perception \& Psychophysics, 1976, 19, 226-230.

Meiselman, H. L., \& Halpern, B. P. Enhancement of taste intensity through pulsatile stimulation. Physiology \& Behavior, 1973, 11, 713-716.

SHIck, T. R. Time course of human gustatory adaptation. Unpublished master's thesis, University of Pittsburgh, 1972.

WEIsZ, P. B. Diffusion and chemical transformation. Science, 1973, 179, 433-440.

(Received for publication November 1, 1977; accepted November $25,1977$. ) 